\title{
Journal of Robotic Surgery: introducing the new publication
}

\author{
Vipul Patel $\cdot$ Christiane Notarmarco
}

Published online: 27 February 2007

(C) Springer London 2007

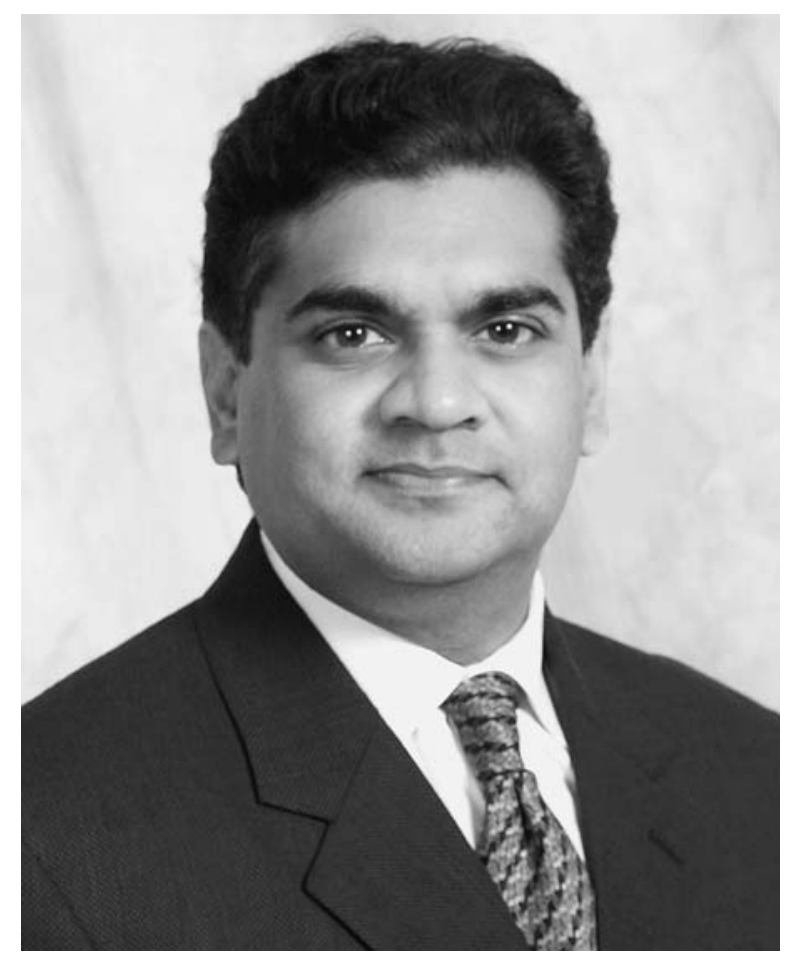

Vipul Patel, MD, Editor-in-Chief

V. Patel $(\bowtie)$

Editor-in-Chief, Division of Urology,

Ohio State University,

538 Doan Hall, 410 West 10th Avenue,

Columbus, OH 43210-1228, USA

e-mail: Patel.914@osu.edu

C. Notarmarco

Executive Editor, Springer-Verlag London Limited,

Ashbourne House, The Guildway, Old Portsmouth Road,

Artington, Guildford GU3 1LP, UK
Welcome to the first issue of the Journal of Robotic Surgery, an exciting new quarterly publication dedicated to robotic applications in surgery.

Surgery has traditionally been a specialty within the medical profession that has revolved around invasive procedures to treat various maladies. Initially, trauma induced by the therapeutic procedure was necessary and reasonable to provide benefit to the patient. But now, through the innovation of digital imaging technology, combined with optical engineering and improved video displays, surgeons can operate inside body cavities for therapeutic intervention without requiring the larger incisions previously necessary to allow a surgeon's hands access to the necessary organs. Rather than creating large incisions several inches long to gain access to underlying tissues, minimally invasive surgical techniques typically rely on small half-inch incisions encircling the surgical field in order to insert small scopes and instruments. Minimally invasive surgery has caused a change in the route of access and has significantly and irrevocably changed the surgical treatment of most disease processes. Patients still undergo interventions to treat disease, but minimally invasive surgery makes possible a reduction or complete elimination of the "collateral damage" required to gain access to the organ requiring surgery. While the benefits of this approach are numerous for the patient, early technology limited the application of minimally invasive surgery to certain procedures. Specifically, surgeons using standard minimally invasive techniques lost the advantages of a natural 3-D image, depth perception, and articulated movements. Magnification of small structures was often difficult, and instruments were rigid and without joints. Robotic surgery has provided the technology to address these limitations and allow the application of minimally invasive surgery to a broader spectrum of patients and their diseases. Surgical robots relieve some of 
these limitations by providing fine motor control, magnified 3-D imaging, and articulated instruments.

In surgery, robots have taken on a different role due to varying demands and applications. Surgical robots must have the capability of a human interface to allow real-time guidance in various alternating scenarios. Modern-day surgical robots are a form of computer-assisted surgery using a "master-slave relationship" in which the surgeon is able to control the actions of the robot in real time, using the robot to improve upon his/her vision, dexterity and overall surgical precision. The most commonly used robotic surgical system is the da Vinci robot (Intuitive Surgical, Sunnyvale, CA, USA).

The birth of this journal is perfectly timed, as the field of robotics has evolved beyond its infancy and has proven itself to be a useful and lasting innovation. The use of robotics in surgery is now broad-based across multiple surgical specialties and will undoubtedly expand over the coming decades as new technical innovations and techniques increase the applicability of its use.

Currently, robotic surgery articles are published in various and diverse surgical journals, but none is seen as the leading resource for robotic surgical education. There is unprecedented enthusiasm worldwide for the application of robotics in surgery, but a void exists in the literary resources to support the growth of this new field. The Journal of Robotic Surgery seeks to fill this void and become the leading worldwide journal for the publication of articles relating to robotic surgery, encompassing surgical simulation and integrated imaging techniques. The journal will provide a centralized, focused resource for physicians wishing to publish their experiences or to acquaint themselves with the most up-to-date findings in the field.

The Journal of Robotic Surgery will publish papers on robotic surgical applications in urology, cardiac surgery, gynecology, ENT, neurosurgery and general surgery. The scope of the journal is extensive and includes original articles, reviews, tips and tricks, techniques, simulation and imaging, and educational training. Articles are enhanced by the addition of electronic supplementary material, whereby the online version includes videos, slides and library resources.

The Journal of Robotic Surgery is available in both electronic and print formats to serve the needs of librarians and readers around the world. It is available via SpringerLink, Springer's fully integrated online platform. Both Online First, a feature where articles are published online before they appear in print, and Editorial Manager, an online peer review and author submission system, are in place for the journal. In addition, thanks to the generous sponsorship of Intuitive Surgical, all articles in Volume 1 will be freely accessible (full text and videos) to all through Springer's Open Choice program.

The journal has a strong international Editorial Board made up of experts from all of the surgical fields. As Editor-in-Chief, I am extremely grateful for the support the board members have given me and for the time and effort they have put into getting the first issue published, by both contributing and reviewing papers. I look forward to working with them in the coming years and making this journal the success that I feel sure it will be.

More information about the journal can be found on its homepage at http://www.springer.com/journal/11701. We invite you to contribute your own work-which will be fully open access upon acceptance and publication-and share your experiences of robotic surgery, via http:// jors.edmgr.com. We welcome not only your contributions but also your comments and suggestions. Together, we feel sure we can make this the journal that you, the readers, want. 